\title{
Capturing and Querying Structural Provenance in Spark with Pebble
}

\author{
Ralf Diestelkämper and Melanie Herschel \\ IPVS - University of Stuttgart \\ Stuttgart, Germany \\ \{ralf.diestelkaemper|melanie.herschel\}@ipvs.uni-stuttgart.de
}

\begin{abstract}
Analyzing and debugging Spark processing pipelines is a tedious task which typically involves a lot of engineering effort. The task becomes even more complex when the pipelines process nested data. Provenance solutions that track the derivation process of individual data items assist data engineers while debugging these pipelines. However, state-of-the-art solutions do not precisely track nested data items.

We demonstrate Pebble, a system for capturing and querying a new type of provenance on nested data in Spark called structural provenance. It captures access and modification of top-level as well as nested data items, and allows querying the provenance of nested items based on tree-patternmatching. Implemented as a standalone library on top of Apache Spark, it seamlessly leverages the underlying infrastructure for scalability. Through the graphical user interface implemented in a Jupyter notebook we showcase ten debugging scenarios of Spark programs on real-world datasets.
\end{abstract}

\section{ACM Reference Format:}

Ralf Diestelkämper and Melanie Herschel. 2019. Capturing and Querying Structural Provenance in Spark with Pebble. In 2019 International Conference on Management of Data (SIGMOD '19), fune 30-fuly 5, 2019, Amsterdam, Netherlands. ACM, New York, NY, USA, 4 pages. https://doi.org/10.1145/3299869.3320225

\section{INTRODUCTION}

Big Data analytics systems such as Apache Hadoop, Flink, and Spark have built-in support for processing nested data sources such as JSON, Protocol Buffers, and XML files. That is

Permission to make digital or hard copies of all or part of this work for personal or classroom use is granted without fee provided that copies are not made or distributed for profit or commercial advantage and that copies bear this notice and the full citation on the first page. Copyrights for components of this work owned by others than ACM must be honored. Abstracting with credit is permitted. To copy otherwise, or republish, to post on servers or to redistribute to lists, requires prior specific permission and/or a fee. Request permissions from permissions@acm.org.

SIGMOD '19, fune 30-fuly 5, 2019, Amsterdam, Netherlands

(c) 2019 Association for Computing Machinery.

ACM ISBN 978-1-4503-5643-5/19/06 ..\$15.00

https://doi.org/10.1145/3299869.3320225

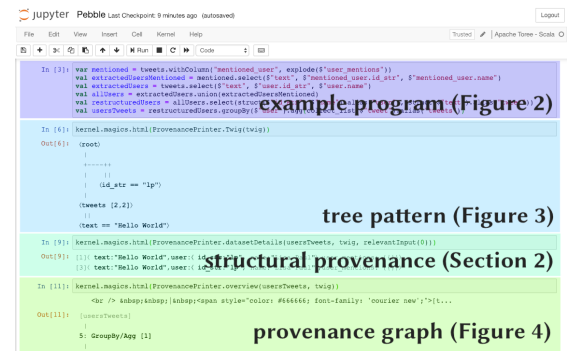

Figure 1: Screenshot of Pebble in a Jupyter notebook

why data engineers frequently choose these systems to process nested data in complex pipelines. However, analyzing and debugging these pipelines is tedious.

We demonstrate Pebble that supports data engineers in analyzing and debugging pipelines in Apache Spark. Pebble implements a novel type of provenance called structural provenance. In general, provenance describes the derivation process of data items [4] all the way from the source data to the result data. In addition to tracing the data itself, structural provenance also traces the access and structural manipulations of nested data items. Pebble collects structural provenance during Spark program execution and offers tree-patterns to query the collected provenance. These allow data engineers to precisely reference nested items and to put them into relation. Pebble implements a novel, scalable tree-pattern-matching (TPM) algorithm to match the treepatterns on the data. To provide an interactive debugging experience, Pebble provides visual representations of the tree-patterns, the matching data, and operators.

Figure 1 shows a screenshot of Pebble, integrated in a Jupyter notebook. Colors highlight different components, which are subsequently discussed in more detail in this paper and the video (https://youtu.be/YUSEydCKqCk). Overall, Pebble implements the following research contributions and, to the best of our knowledge, demonstrates, for the first time, scalable provenance capture and querying for nested data.

Structural provenance capture. Pebble captures structural provenance for nested data items. Structural provenance records the access and manipulation of each potentially nested data item during processing. In the context of big data analytics, it is the first provenance approach that explicitly distinguishes between the access of a data item, 
e.g., filtering of a value, and the manipulation of a data item, e.g., unnesting of a nested collection, or the re-assignment of a value. This is required to precisely and effeciently resolve data dependencies of nested items.

Tree-pattern-matching query. Existing Big Data analytics solutions provide no means to query individual nested data items. In order to address individual items or hand-picked combinations of them, Pebble implements treepatterns and tree-pattern-matching [8] on top of Spark. To the best of our knowlege, it is the first solution to provide a distributed and scalable tree-pattern-matching solution.

Interactive exploration. In order to deliver an interactive exploration and debugging experience, Pebble is integrated in a Jupyter Notebook. It provides provenance views for programs, operators, and dataframes, which precisely visualize attributes relevant to a scenario under observation.

Related work. To put the above contributions in perspective to existing provenance solutions for Big Data analytics systems like Spark, we briefly review related work. Titian [6], Newt [7], and RAMP [5] only provide data provenance of flat data items. In contrast, Pebble provides structural provenance that suits both flat and nested data items. The only provenance solution supporting nested data items is Lipstick [1], which is only capable of tracing data values. In constrast, Pebble traces data values and data structures and offers a scalable solution by not relying on an in-memory provenance graph. In addition, Pebble is the only solution that offers a systematic, tree-pattern-based approach to query provenance based on items structure, e.g., the nesting order of attributes or the amount of nested lists.

Structure. Section 2 walks through an example debbugging session to show Pebble's features. In Section 3, we show how Pebble's architecture transparently integrates in Spark. Section 4 covers the audience experience.

\section{EXAMPLE SCENARIO}

Let us consider a use case on Twitter data. An excerpt of the data, used as input in our example, is shown in Table 1. Our program groups tweets by distinct users, considering both tweets that users authored and tweets they were mentioned in (Figure 2). The result is shown in Table 2. We observe that the text Hello World occurs twice in the nested list of user Lisa Paul. To find the cause for the duplicate, we debug the the program in a Pebble-enabled Jupyter Notebook.

Capture. To capture structural provenance, we simply replace Spark's read method with Pebble's Provenance.readJson(...) method (Figure 2, l. 1). It triggers Pebble to associate a unique identifier to each processed top-level data item. Pebble also records which attributes an operator accesses and how an operator modifies an input item to contribute to the output. When processing

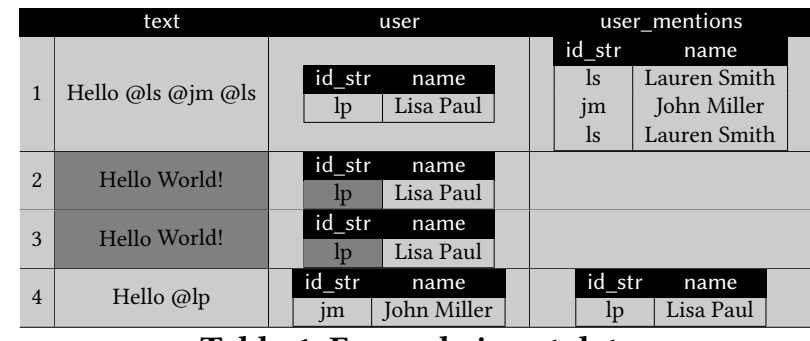

Table 1: Example input data

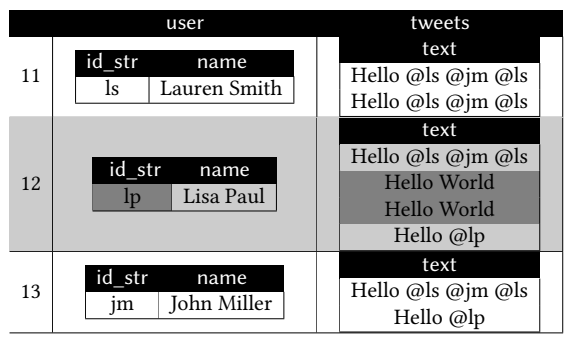

Table 2: Example result data

nested collections, Pebble further keeps track of positions within the list, e.g., when the program flattens the nested user_mentions collection (Figure 2, l. 2). These recordings are based on inference rules defining the structural provenance. Similarly to [3], they extend operator semantics of the nested relational calculus.

Querying. Once Pebble has computed the program result - and the provenance behind the scenes - it can support data engineers in expressing their information need to explain the previously mentioned duplicate in form of a tree-pattern. Indeed, Spark does not provide built-in means to address individual items in nested collections.

A tree-pattern consists of nodes representing attributes in the schema and edges representing relationships between them [8]. Edges represent parent-child (PC) or ancestordescendant $(\mathrm{AD})$ relationships. The relationships impose structural constraints over the schema, because the treepattern only matches the result if all PC and AD relationships are satisfied. In addition, we may also associate nodes with value and cardinality constraints. Value constraints are satisfied if the value in the data matches the value associated with a tree-pattern node. Cardinality constraints define how many matching descendant nodes must be in the data. They are only applicable on nodes that match nested collections.

In our example, we define the tree-pattern visualized in Figure 3. It references the attribute text that has to be a descendant of the tweets attribute to satisfy the structural constraints. Further, it must have the value Hello World to satisfy the value constraint and it must occur twice to satisfy the cardinality constraint of the tweets node. Pebble's scalable tree-pattern matching algorithm matches this tree-pattern to the dark-grey items in Table 2. 


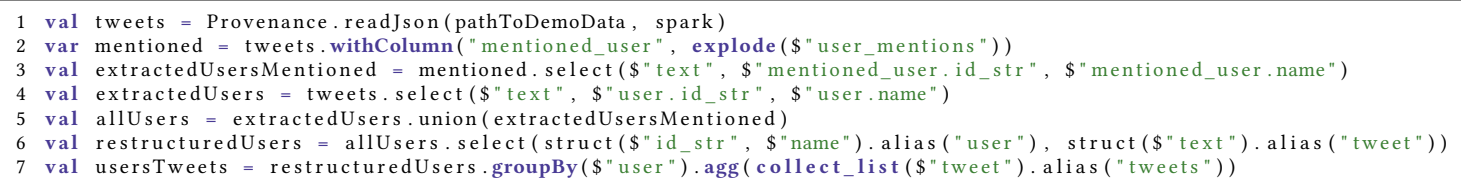

Figure 2: Example program that collects all tweets of an authoring or mentioned user in a nested collection

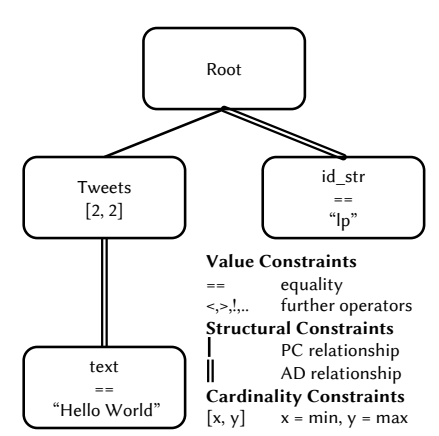

Figure 3: Tree-pattern that matches the dark-grey items in Table 2

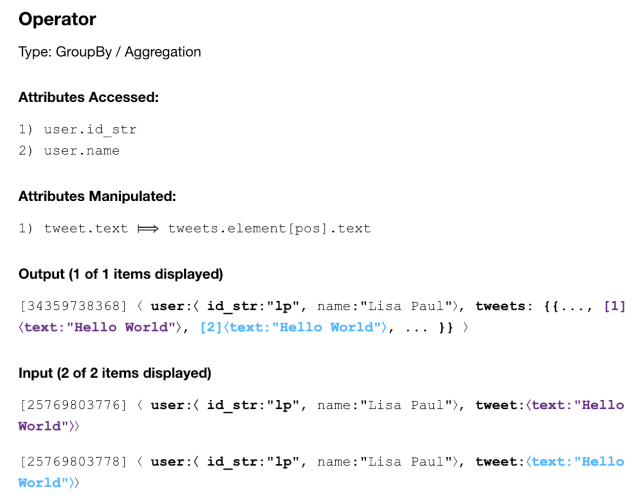

Figure 5: Operator details for groupby and aggregation

Backtracing. As entry point to the backtracing, Pebble provides a Provenance.structuralBacktrace (...) method for matching the tree-pattern on the result and tracing the matched items back to the input. After backtracing, Pebble yields the dark-grey items in the input Table 1. They explain how the queried duplicate was obtained: Lisa Paul has tweeted Hello World twice.

Comparison to data provenance. In comparison, data provenance solutions, such as Titian [6], trace back the entire light-grey item of the result (Table 2) to the four light-grey items of the input (Table 1). Imagine Lisa has tweeted another hundreds of texts and got mentioned in few thousand tweets more. All these tweets would have contributed to the provenance of the light-grey result item. Further notice that the data provenance consists of entire tweets. In our real world dataset these have roughly 1000 attributes. Consequently, existing data provenance solutions would overwhelm the data engineer with "imprecise" data provenance, consisting of a large number of wide data items. Note that in contrast, whereprovenance [2] (a different kind of data provenance than the data provenance supported by data analysis systems) would return too little information to explain the duplicate. Details are omitted due to space constraints. Opposed to existing solutions, Pebble allows to precisely pinpoint relevant nested source data items by leveraging structural provenance.

Provenance graph. To further improve the debugging experience in the Jupyter Notebook, Pebble offers a compact summary of the provenance, called provenance graph. Figure 4 shows the provenance graph of our running example. It consists of the program's operator tree annotated with the number of top-level items traced back to the input (in square brackets). In our example, the graph reveals that no item went through the flatten operator. Consequently, no tweet that mentions Lisa Paul contributed to the result.

Detail views. Investigating the input data and the provenance graph may not be sufficient to identify unintended data manipulations. For that purpose, Pebble offers a detail view for each operator. Figure 5 shows the operator details of the groupby and aggregation operation. It reveals that the nested user attributes are accessed, but not manipulated unlike the tweets.text attribute. It becomes member of the nested tweets collection and gets a unique position. Thereby, Pebble can precisely track just the colored text items in Figure 5.

In conclusion, Pebble's structural provenance implementation allows to precisely track and query nested data items in Spark programs for debugging purposes.

\section{PEBBLE'S ARCHITECTURE}

Pebble extends Apache Spark to provide the functionality described in Section 2. It also provides an HTML printer to generate output in the web-based notebooks. In Figure 6, we show Pebble's modules (blue) on top of Spark (black), embedded in an Apache Toree kernel that runs in a Jupyter Notebook (grey). In the following, we sketch the modules' internals.

Provenance capture. The Pebble module consists of two submodules. The first one is the Capture module. It extends Spark's distributed dataframes and operators. The 


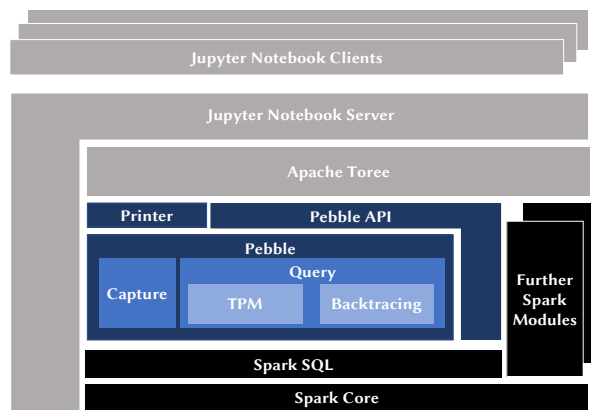

Figure 6: Pebble's architecture

dataframes are bags of items that share the same schema and allow for nested bags and sets. The module associates a unique identifier to each top-level item in the dataframe. In addition, the Capture module extends and, thus, collects provenance for the following Spark operators: read, select, filter, join, union, groupby, aggregation, map with UDFs, flatten and nested queries. In case of a groupby, aggregation, or flatten operator, the module also records positions of the nested items being manipulated.

Provenance query. The Query module is Pebble's second submodule. It is responsible for querying the captured provenance and consists of a Backtracing and a Tree-PatternMatching (TPM) module.

The TPM module implements tree-patterns and a Sparktailored tree-pattern-matching algorithm, which has two major phases. Opposed to existing approaches that focus on semi-structured XML data [8], Pebble exploits the fact that Spark internally considers a structured nested data model. During the first phase, it matches the structure of the treepattern against the schema of the result dataframe. It yields a set of schema matches, which fulfill the structural constraints. During the second phase, the algorithm generates a filter statement that calls a user-defined function for each top-level item in the dataframe. The user defined function checks for each item whether it fulfills all structural, value, and cardinality constraints of the tree-pattern. The explicit seperation into a schema matching and a data matching phase let the implementation scale with the Spark-cluster unlike other state-of-the-art approaches that rely on global data structures [8].

The Backtracing module implements the means to trace back items that match the tree-patterns. It joins the identifiers captured during program execution until the input is reached. The module extends Titian's backtracing on item identifiers [6] with conditions reflecting relevant nested data items, e.g., the positions of items in nested collections.

Pebble API. The Pebble API module masks the Spark extensions of the previous modules so that programming with Pebble looks and feels like standard Spark programming. It is the entry point to capturing and querying the provenance.
Printer. The Printer module provides the HTML views mentioned in the previous section. It requires an HTML renderer from a Jupyter or any other kind of notebook.

Architecture comparison. Pebble is a standalone library that does not require any modification of Spark's provided modules unlike existing solutions. Our experiments indicate that it is binary compatible between versions 2.1 (2016) and 2.4 (2019). Since Pebble only requires an HTML printer, it runs on Jupyter Notebooks with Apache Toree kernels or Almond-Spark kernels, and on Apache Zeppelin Notebooks. Thus, it runs in a wide variety of environments.

\section{AUDIENCE EXPERIENCE}

We run Pebble on a remote Spark cluster of six computing nodes. The cluster stores two real-world datasets: a 2 GB DBLP dataset (https://dblp.org/) and a 500 GB dataset containing Twitter tweets (https://developer.twitter.com/). For each of these datasets, we have prepared five debugging scenarios that include a Spark program and a tree-pattern expressing a data engineer's debugging interest. Besides demonstrating these prepared scenarios, we invite attendees to define their own programs and provenance questions.

\section{CONCLUSION}

This demonstration presents Pebble, a system that tracks structural provenance of nested data in Big Data analytics pipelines. This provenance can be queried using treepatterns. These capabilities are integrated in a Jupyter notebook that supports data engineers in debugging their Big Data analytics pipelines. We demonstrate these features on ten different scenarios over two real-world datasets.

Acknowledgements. Partially supported by the Deutsche Forschungsgemeinschaft (DFG, German Research Foundation) under Germany's Excellence Strategy - EXC 2120/1 - 390831618 and TRR $161-251654672$.

\section{REFERENCES}

[1] Y Amsterdamer, S B Davidson, D Deutch, T Milo, J Stoyanovich, and V Tannen. 2011. Putting Lipstick on Pig: Enabling Database-style Workflow Provenance. PVLDB 5, 4 (2011).

[2] P Buneman, S Khanna, and W-C Tan. 2001. Why and Where: A Characterization of Data Provenance. In ICDT.

[3] M Grabowski, J Hidders, and J Sroka. 2013. MapReduce optimisation in the Nested Relational Calculus. In BNCOD.

[4] M Herschel, R Diestelkämper, and H Ben Lahmar. 2017. A survey on provenance: What for? What form? What from? VLDB f. 26, 6 (2017).

[5] R Ikeda, H Park, and J Widom. 2011. Provenance for Generalized Map and Reduce Workflows. In CIDR.

[6] M Interlandi, A Ekmekji, K Shah, M A Gulzar, S D Tetali, M Kim, T D Millstein, and T Condie. 2018. Adding data provenance support to Apache Spark. VLDB J. 27, 5 (2018).

[7] D Logothetis, S De, and K Yocum. 2013. Scalable lineage capture for debugging DISC analytics. In SoCC.

[8] J Lu, T W Ling, Z Bao, and C Wang. 2011. Extended XML Tree Pattern Matching: Theories and Algorithms. TKDE 23, 3 (2011). 